Accepted for publication: Astronomische Nachrichten, (AN), 2013, 334, p. 73 (Cambridge Workshop 17 on Cool Stars, Stellar Systems and the Sun)

\title{
New insights: the accretion process and variable wind from TW $\mathrm{Hya}^{1}$
}

\author{
A. K. Dupree \\ Harvard-Smithsonian Center for Astrophysics, 60 Garden Street, Cambridge, MA 02138 \\ USA \\ dupree@cfa.harvard.edu
}

\begin{abstract}
For the first time in a classical $\mathrm{T}$ Tauri star, we are able to trace an accretion event signaled by an hour-long enhancement of X-rays from the accretion shock and revealed through substantial sequential changes in optical emission line profiles. Downflowing turbulent material appears in $\mathrm{H} \alpha$ and $\mathrm{H} \beta$ emission.

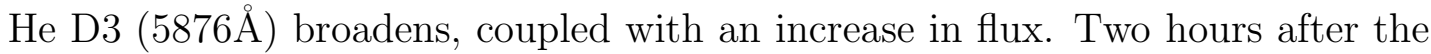
$\mathrm{X}$-ray accretion event, the optical veiling increases due to continuum emission from the hot splashdown region. The response of the stellar coronal emission to the heated photosphere follows about 2.4 hours later, giving direct evidence that the stellar corona is heated in part by accretion. Then, the stellar wind becomes re-established. A model that incorporates the dynamics of this sequential series of events includes: an accretion shock, a cooling downflow in a supersonically turbulent region, followed by photospheric and later, coronal heating. This model naturally explains the presence of broad optical and ultraviolet lines, and affects the mass accretion rates currently determined from emission line profiles. These results, coupled with the large heated coronal region revealed from X-ray diagnostics, suggest that current models are not adequate to explain the accretion process in young stars.
\end{abstract}

Subject headings: stars: individual (TW Hya) - stars: mass-loss - stars: variables: T Tauri stars - stars: winds, outflows - X-rays: stars

\footnotetext{
${ }^{1}$ Data were obtained with the CHANDRA satellite, the $6.5 \mathrm{~m}$ Magellan/Clay telescope at Las Campanas Observatory, and Gemini-S which is operated by the Association of Universities for Research in Astronomy, Inc. under a cooperative agreement with the US-NSF on behalf of the Gemini partnership.
} 


\section{Introduction}

TW Hya (CD -34 7151; HIP 53911) is arguably the closest accreting T Tauri star making it a choice object for detailed spectroscopic study. It is generally thought that cool material from the circumstellar disk surrounding the star is channeled by the stellar magnetic field and free-falls towards the star where a shock forms. The X-ray and optical spectra reported here probe this accretion process. Another major attribute of TW Hya is the fact that its surrounding circumstellar disk appears roughly face-on (Qi et al. 2004) so that the critical polar regions, where the accretion process occurs, can be observed directly without obscuration by the disk.

A major observational campaign was aimed towards TW Hya that involved dedicated X-ray spectroscopic observations amounting to 500 total ks with the CHANDRA spacecraft. These results are discussed in Brickhouse et al. (2010). Contemporaneous optical and infrared spectroscopy and photometry were carried out from 4 continents (Dupree et al. 2012). As a result, the process of accretion can be investigated, and for the first time, the source of the broad emission lines from the star can be reliably identified.

\section{X-ray accretion signatures}

The CHANDRA X-ray spectra contain many emission lines arising from high temperatures. Analysis of the line strengths reveals that three different components producing X-rays must be present in the TW Hya atmosphere: a high temperature $\left(\sim 10^{7} \mathrm{~K}\right)$ corona, a lower temperature $\left(3 \times 10^{6} \mathrm{~K}\right)$ component arising from the accretion shock, and a large volume producing $\mathrm{O}$ VII, at slightly lower temperature $\left(2.5 \times 10^{6} \mathrm{~K}\right)$ with density lower than the accretion shock itself (Brickhouse et al. 2010). The behavior of lines arising in the accretion shock, namely N VII, O VIII, Ne IX, Fe XVII, and Mg XI, can give a direct measure of the instantaneous strength of the accreting material. These lines are marked in the CHANDRA spectrum shown in Fig 1, and the variation of the strength of the sum of the accretion lines is shown in Fig. 2

The accretion line flux (Fig. 2) reveals an enhancement centered at JD 2454157.75 that represented the highest count rate in a $3 \mathrm{ks}$ average of the long 500ks CHANDRA observation, and merits special study. 


\section{Simultaneous optical spectra}

Many simultaneous high-resolution optical spectra were taken during the X-ray accretion enhancement, providing the opportunity to evaluate the effect of the increase in the accreting line flux on the optical emission lines. Echelle spectra were taken continuously over 3 nights with the MIKE spectrograph at the Magellan/CLAY telescope of Las Campanas Observatory. These spectra have a resolution $\sim 35,000$ so that the line profiles are well-resolved. Fig. 3 shows the behavior of the total flux and the line profile asymmetries during the span of the X-ray measures shown in Fig. 2. The flux of $\mathrm{H} \alpha$ does not exhibit any systematic change, which is not surprising since the line is surely optically thick. However, the asymmetry of the line does change quite abruptly following the X-ray accretion event. The asymmetry of a line profile indicates the mass flow in a differentially moving line-forming region (Hummer \& Rybicki 1968). Of course, if a slab moves at constant velocity, the whole profile will shift by an amount corresponding to the constant velocity. But differential motion as found in a stellar wind or in downflowing material produces a change in the line asymmetry. If the short-wavelength side ("blue") of the line is stronger than the long-wavelength side ("red"), material is flowing away from the observer and vice versa. The abrupt increase in the value of the blue:red ratio for $\mathrm{H} \alpha$ suggests an abrupt increase in down-flowing material that begins about 9 minutes after the increase in the X-ray accretion line flux. This increased inflow continues for about 1.5 hours. The $\mathrm{H} \beta$ emission line exhibits a similar increase in the ratio of blue to red emission, echoing the increased downflow of material exhibited by the $\mathrm{H} \alpha$ line. In addition, an increase in the strength of the total $\mathrm{H} \beta$ emission occurs.

The D3 line of He I at $5876 \AA$ is also a valuable probe of the accretion process. It is known to have both a broad and narrow component of emission. The broad component is generally thought to signal accretion (Donati et al. 2011) in a similar way as the broad lines of the Balmer series. A sharp increase in the flux at the end of the X-ray accretion event results from an enhanced long wavelength wing of the line profile, and the increase in line flux beginning about 30 minutes later (see Fig. 4) is due to a $30 \%$ increase in the broad component of the line, whereas the narrow component is constant to within $15 \%$. The sequential changes of the optical lines following the X-ray accretion strongly suggests that they form in the post-shock cooling zone.

The line widths themselves give additional support to this formation scenario. The Balmer lines have a FWHM of about $\pm 150 \mathrm{~km} \mathrm{~s}^{-1}$, which is obviously in excess of a thermal width $\left(\sim 21 \mathrm{~km} \mathrm{~s}^{-1}\right)$ at a temperature of $10^{4} \mathrm{~K}$, and also in harmony with the measured widths of the Ne IX $\left( \pm 165 \mathrm{~km} \mathrm{~s}^{-1}\right)$ lines observed in the CHANDRA spectra (Brickhouse et al. 2010). Far ultraviolet line widths of C III and O VI, when corrected for wind absorption, 
suggest similar line widths of $\pm 160 \mathrm{~km} \mathrm{~s}^{-1}$ (Dupree et al. 2005).

The veiling or 'weakening' of absorption lines in the optical spectrum arises from a continuum and perhaps a contribution from line emission (Petrov et al. 2011) produced by the accretion hot spot in the photosphere. The value of the veiling from the short wavelength

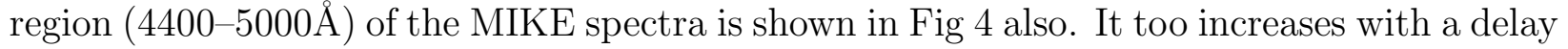
of 2 hours after the X-ray accretion event. Such a delay is consistent with a reasonable size of the photospheric 'hot spot'. Using a value of $35 \mathrm{~km} \mathrm{~s}^{-1}$ for the post shock downflow indicated by substantial absorption at that velocity in the $\mathrm{H} \beta$ profile suggests that material will traverse a distance comparable to the size of a hot spot covering $\sim 10 \%$ of a star with radius $0.8 R_{\odot}$ in 2.8 hours. The flux from the corona (represented by the first order CHANDRA spectrum) responds $\sim 2$ hours later to an increase in veiling (Dupree et al. 2012).

\section{The stellar wind}

The Balmer profiles also reveal the wind structure and its variation. $\mathrm{H} \alpha$ and $\mathrm{H} \beta$ profiles observed over 4 successive nights are shown in Fig. 5 and Fig. 6. The X-ray accretion event discussed earlier occurred during the first night when the $\mathrm{H} \alpha$ profile is roughly symmetric. During the subsequent 3 nights, absorption appears and systematically increases on the negative velocity side of the line. The wind which appears very weak or perhaps absent on the first night, recovers and becomes more opaque during the following nights.

The symmetry of the broad $\mathrm{H} \alpha$ profile on the first night demonstrates that the line is not formed in an accretion stream (which should appear only at positive velocities), but most likely in a turbulent region of the atmosphere with velocity centered on the TW Hya itself. $\mathrm{H} \alpha$ has a higher opacity than $\mathrm{H} \beta$ and would be formed higher in the atmosphere ('at the edges') of a turbulent region than the $\mathrm{H} \beta$ transition. Thus it is not surprising that the inflow signature is stronger in the weaker $\mathrm{H} \beta$ line.

Because the wind also substantially modifies the emission line profiles, this suggests that the line width may not be a good indicator of the accretion rate as has been proposed (Natta et al. 2004). In fact, in our observations, the wind (and undoubtedly accretion contributes also) changes the width of the line at the $10 \%$ level which, if dependent only on accretion, corresponds to a factor of 5 in the mass accretion rate.

The near-infrared He I transition at $\lambda 10830$ has proved to be an excellent tracer of winds from T Tauri stars (Dupree et al. 2005). This transition arises from a metastable level of neutral helium and thus maintains a relatively high population which can absorb the strong

infrared continuum from a cool star. The broad emission appears likely to arise also in the 
post-shock cooling zone of TW Hya, and the extent of the absorption (to $\sim-300 \mathrm{~km} \mathrm{~s}^{-1}$ ) clearly documents the presence of a fast wind. The terminal velocity of the wind varies with time $\left(-260 \mathrm{~km} \mathrm{~s}^{-1}\right.$ in 1992, Dupree et al. $2005 ;-330 \mathrm{~km} \mathrm{~s}^{-1}$ in 2002, Edwards et al. 2006; $-300 \mathrm{~km} \mathrm{~s}^{-1}$ in 2007). Lines formed at even higher kinetic temperatures (O VI and C III) also give evidence of similar high outflow velocities (Dupree et al. 2005) suggesting that this hot wind may be powered as a result of the accretion process which acts as a source of energy and momentum in the upper atmosphere (Matt et al. 2012; Cranmer 2009).

\section{A new model}

These observations reveal a distinct new view of the accretion process in classical $\mathrm{T}$ Tauri stars and suggest that the current models need revision. It has been common to attempt modeling of the emission features in the spectrum as arising from the accretion stream that is channeled by the magnetic field as it approaches free-fall velocity forming an accretion shock (Muzerolle et al. 2000; Kurosawa et al. 2011). The spectra shown here suggest that an accretion event (observed in X-rays) instigated a cascade of changes to the optical emission line profiles. The emission lines arise in the post-shock cooling volume, and the line widths from X-ray profiles (Brickhouse et al. 2010), far UV (Ardila et al. 2002), the optical and the near-IR helium line shown here are all commensurate and broad. Their breadth has been difficult to interpret in the framework of accreting streams (Ardila et al. 2002) or an accretion shock (Lamzin et al. 2007). A turbulent post-shock cooling zone offers the likely solution to the puzzle of the broad profiles, and is supported by the behavior of the optical lines. The emission measure of the post-shock cooling zone exceeds that of the accretion stream by a factor of 100 (Dupree et al. 2012). Another component of the postshock cooling process is the discovery from the O VII diagnostics in CHANDRA spectra (Brickhouse et al. 2010) of a large coronal region with 300 times the volume and 30 times the emission measure of the accretion shock. All of these observations call for a reassessment of current models of accretion in young stars. It appears that the accretion process can both heat the corona, cause turbulent broadening of the emission lines, and provide a means to power an accretion-driven stellar wind. 


\section{REFERENCES}

Ardila, D. R., Basri, G., Walter, F. M., Valenti, J. A., Johns-Krull, C. M. 2002, ApJ, 566, 1100

Brickhouse, N. S., Cranmer, S. R., Dupree, A. K., Luna, G. J. M., Wolk, S. 2010, ApJ, 710, 1835

Cranmer, S. R. 2009, ApJ, 706, 824

Donati, J.-F., Gregory, S. G., Alencar, S. H. P., et al. 2011, MNRAS, 417, 472

Dupree, A. K., Brickhouse, N. S., Cranmer, S. R., et al. 2012, ApJ, 750, 73

Dupree, A. K., Brickhouse, N. S., Smith, G. H., Strader, J. 2005, ApJ, 625, L131

Edwards, S., Fischer, W., Hillenbrand, L., Kwan, J. 2006, ApJ, 646, 319

Hummer, D. G., Rybicki, G. B. 1968, ApJ, 153, L107

Kurosawa, R., Romanova, M. M., Harries, T. J. 2011, MNRAS, 416, 2623

Lamzin, S. A., Romanova, M. M., Kravtsova, A. S. 2007, in J. Bouvier, I. Appenzeller (eds.), Star-Disk Interaction in Young Stars, IAU Symp. 243 , p. 115

Matt, S. P., Pinzón, G., Greene, T. P., Pudritz, R. E. 2012, ApJ, 745, 101

Muzerolle, J., Calvet, N., Briceño, C., Hartmann, L., Hillenbrand, L. 2000, ApJ, 535, L47

Natta, A., Testi, L., Muzerolle, J., Randich, S., Comerón, F., Persi, P. 2004, A\&A, 424, 603

Petrov, P. P., Gahm, G. F., Stempels, H. C., Walter, F. M., Artemenko, S. A. 2011, A\&A, 535, A6

Qi, C., Ho, P., Wilner D., et al 2004, ApJ, 616, L11 


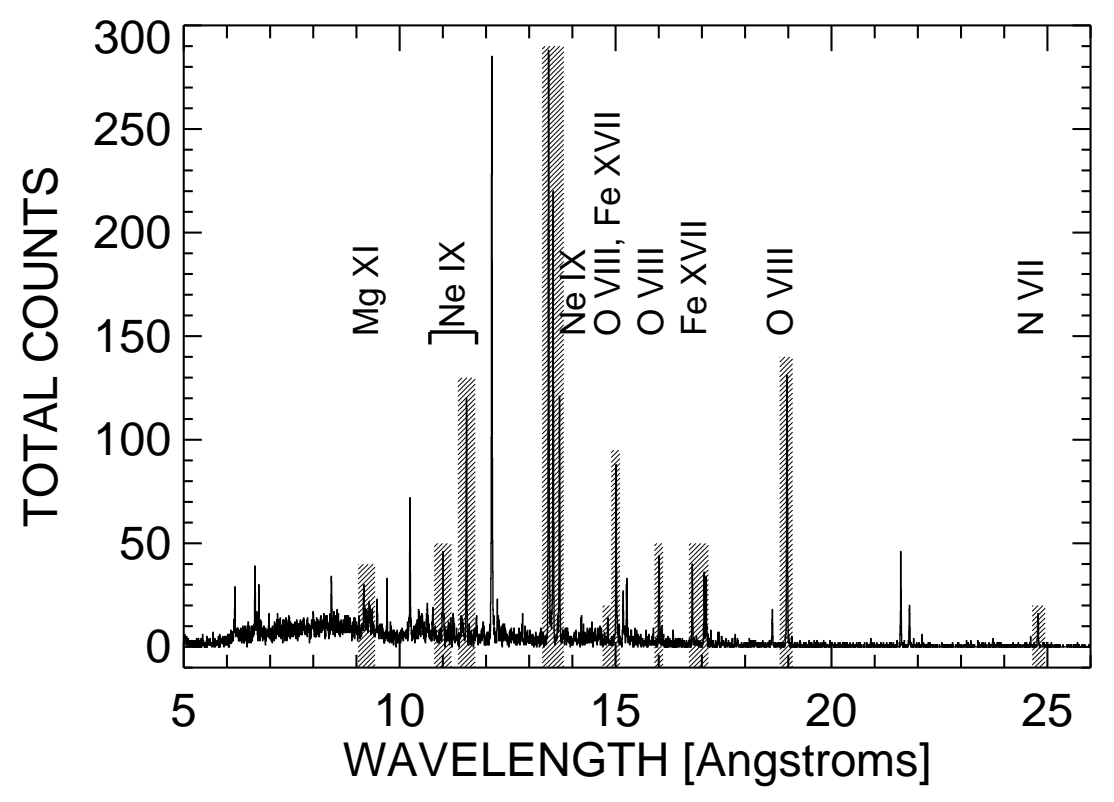

Fig. 1.- Lines arising in the accretion shock (N VII, O VIII, Ne IX, Fe XVII, Mg XI) marked in the MEG total spectrum (hatched areas). 


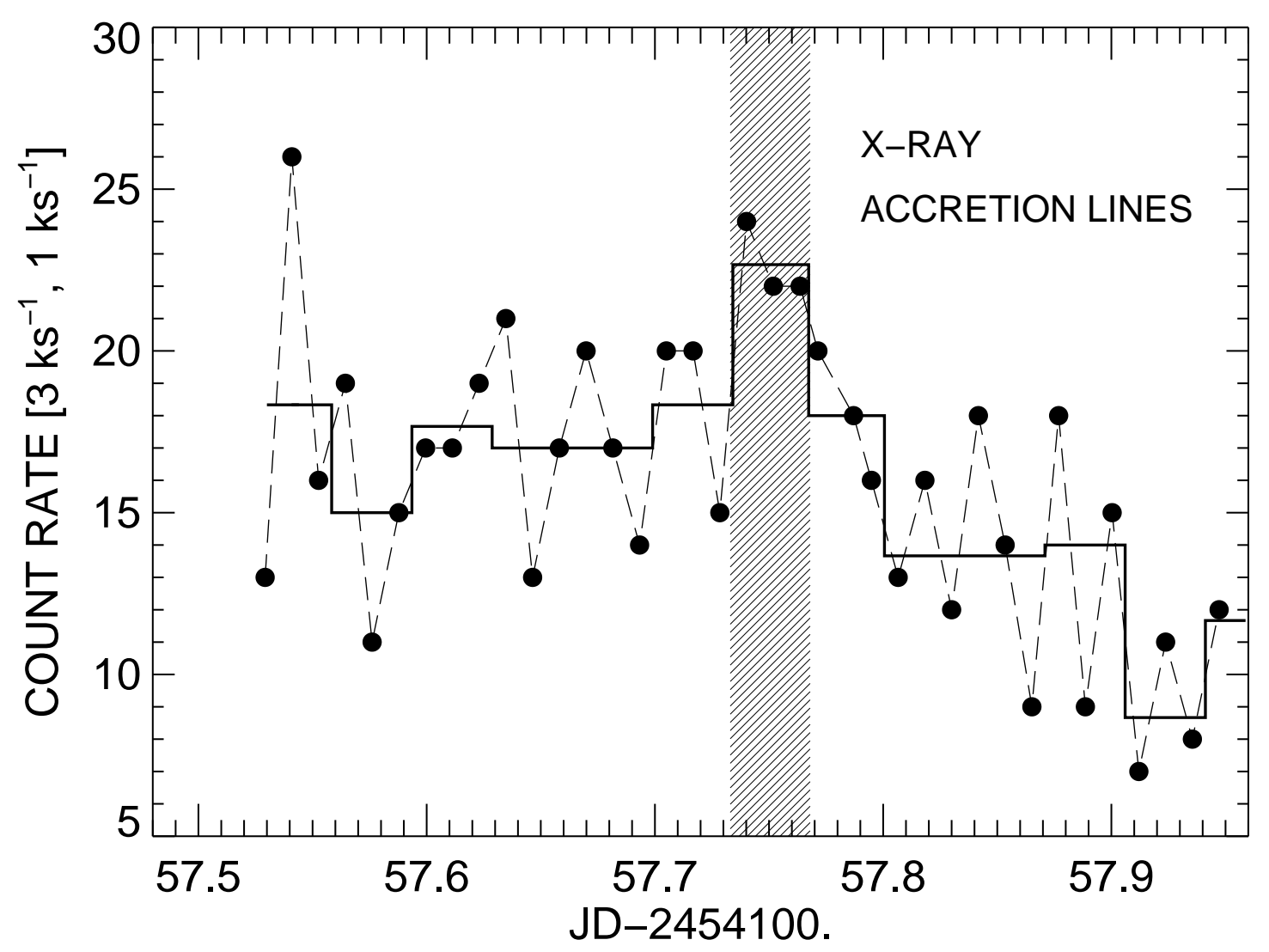

Fig. 2.- Accretion lines (N VII, O VIII, Ne IX, Fe XVII, Mg XI) binned over 1 ks (filled circles) or $3 \mathrm{ks}$ and divided by 3 for display (solid line). The hatched region marks the accretion event and is carried forward in the following figures. 


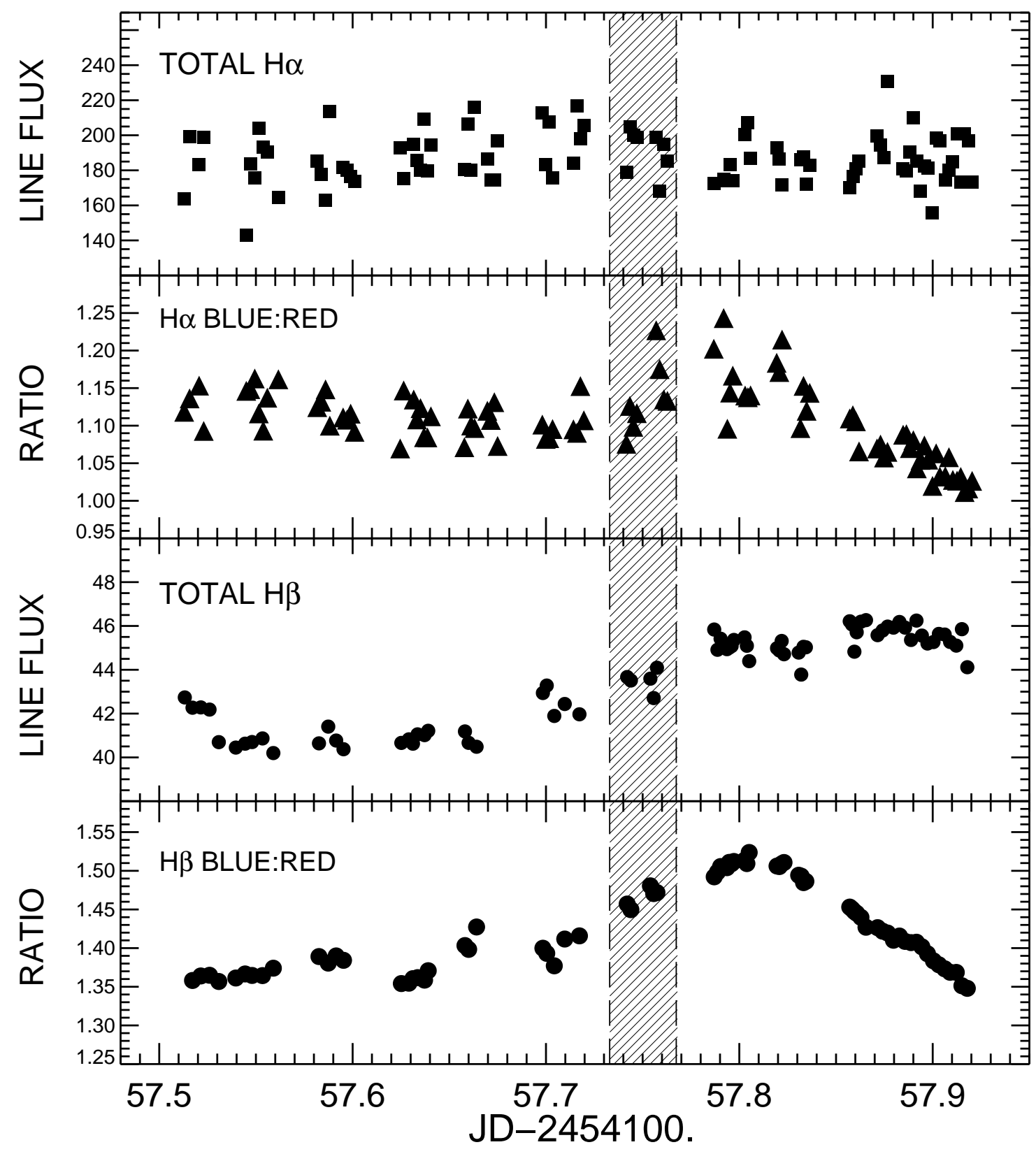

Fig. 3.- The behavior of the $\mathrm{H} \alpha$ and $\mathrm{H} \beta$ line fluxes and profiles. The X-ray accretion event is marked by line hatching. The ratio "blue:red" denotes the flux in $1 \AA$ bandpasss positioned $-2 \AA$ ("blue") or $+2 \AA$ ("red") from line center in continuum-normalized spectra. 


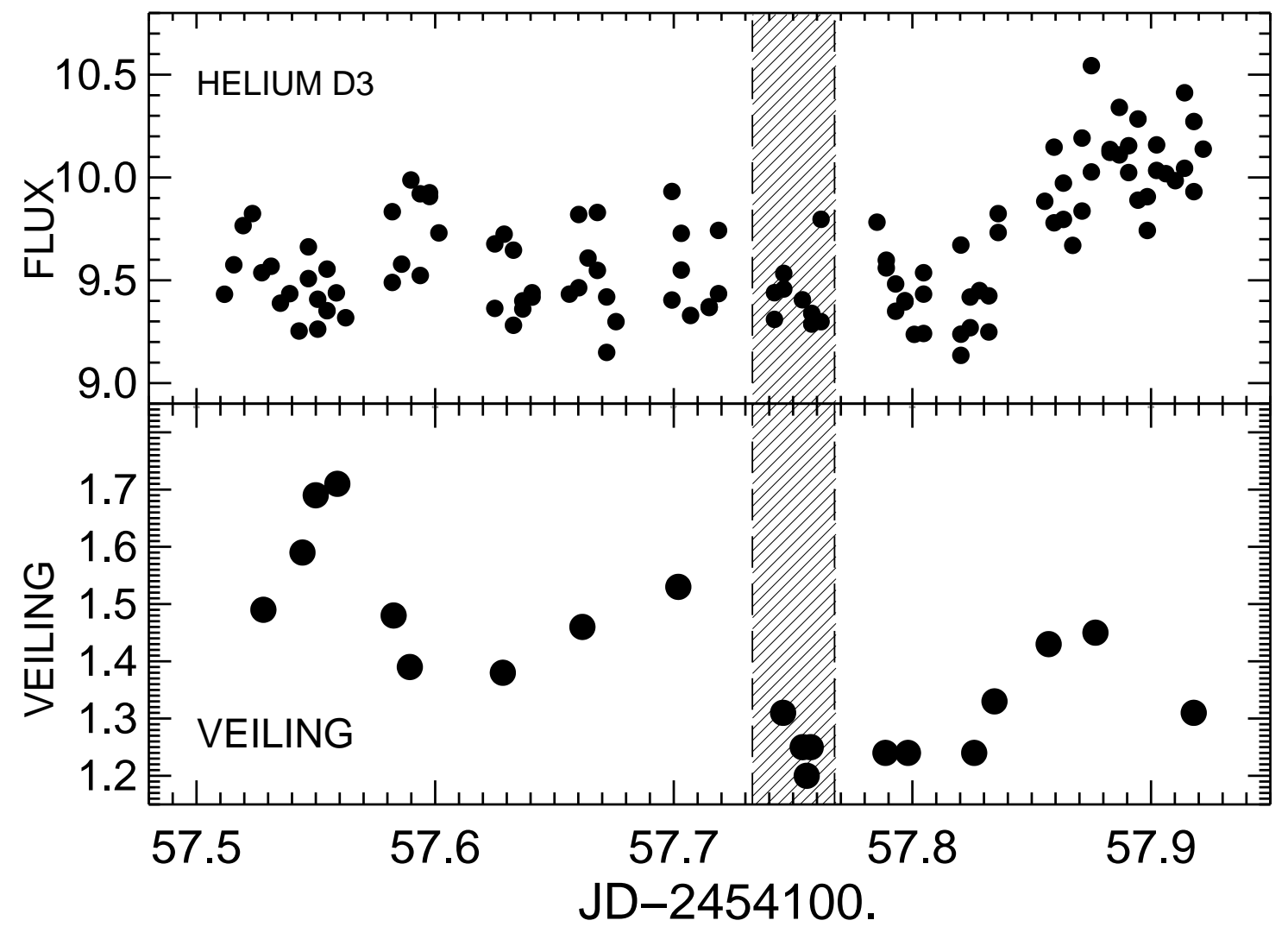

Fig. 4.- The behavior of the He I $5876 \AA$ (D3) flux and the value of the average blue veiling derived from the MIKE spectra. 


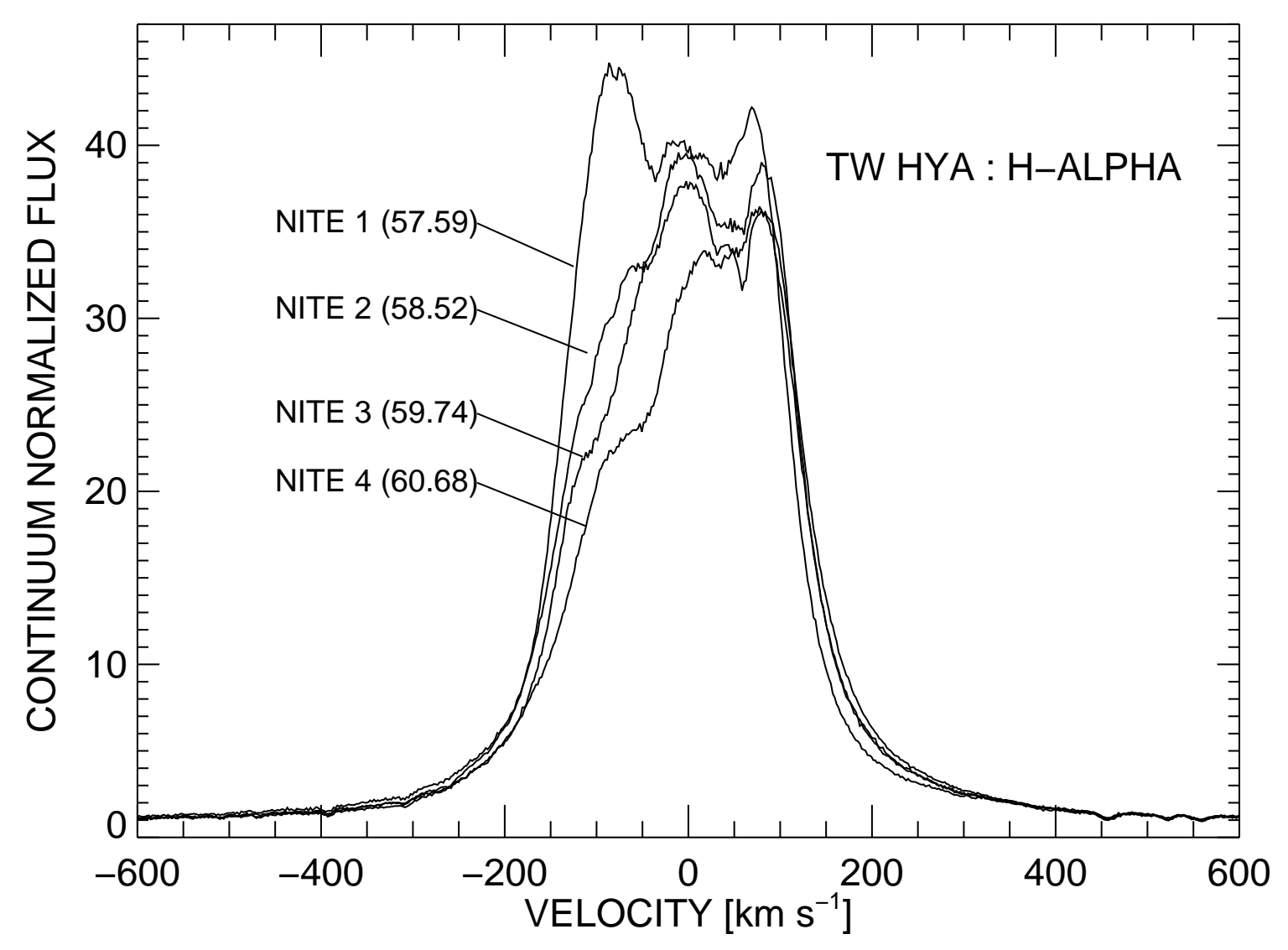

Fig. 5.- The $\mathrm{H} \alpha$ profile over 4 successive nights. Note the systematic apparent onset and strengthening of the wind absorption on the negative velocity side of the profile. The Julian date of each spectrum is marked as JD-2454100. These profiles were taken from the red side spectra of MIKE. 


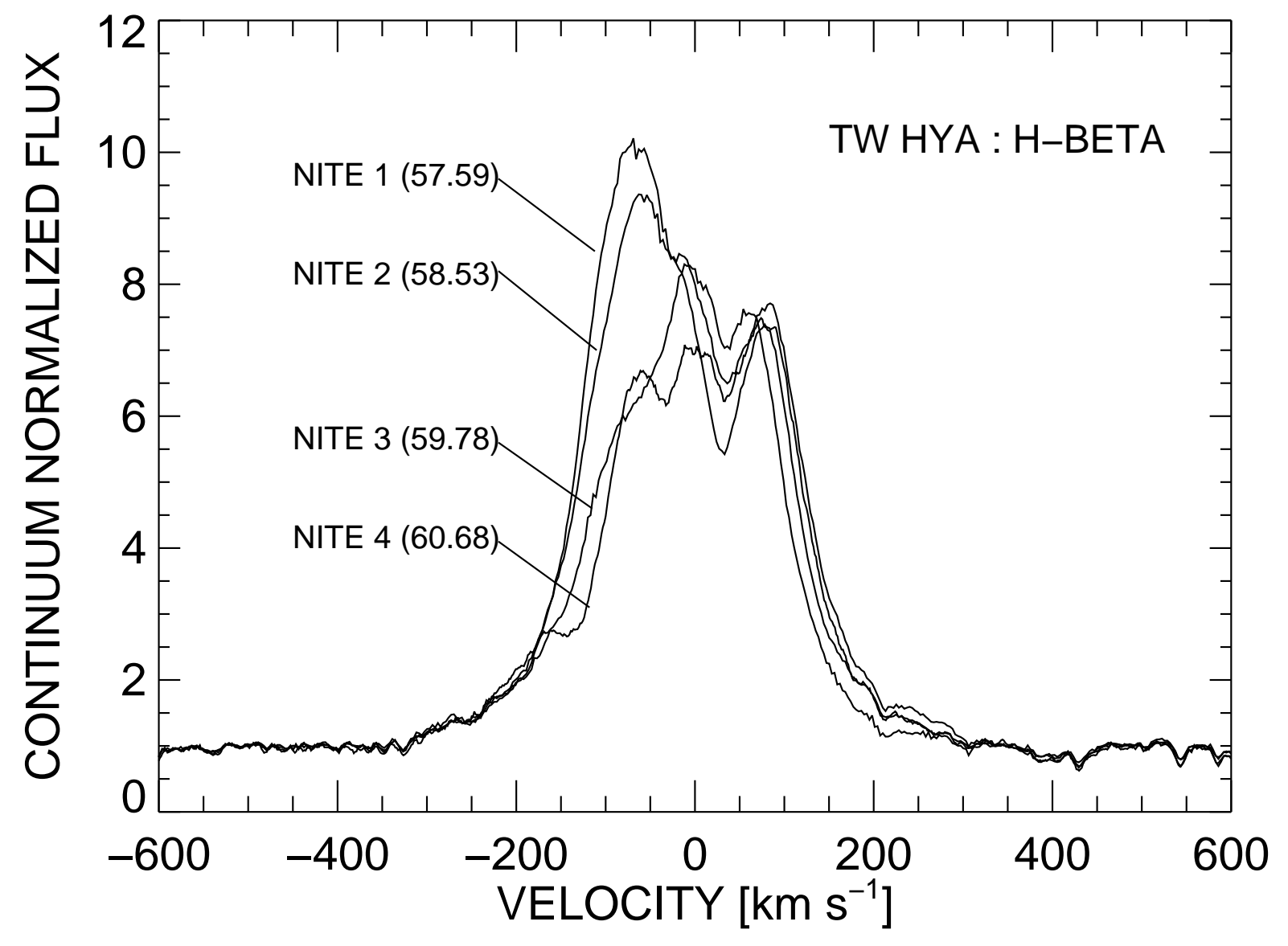

Fig. 6.- The $\mathrm{H} \beta$ profile over 4 successive nights. Note the similarity to $\mathrm{H} \alpha$ with the onset and strengthening of wind absorption. $\mathrm{H} \beta$ exhibits a clear infall signature with absorption at a velocity of $\sim+35 \mathrm{~km} \mathrm{~s}^{-1}$. These profiles were taken from the blue side spectra of MIKE. The Julian date of each spectrum is marked as JD-2454100. 


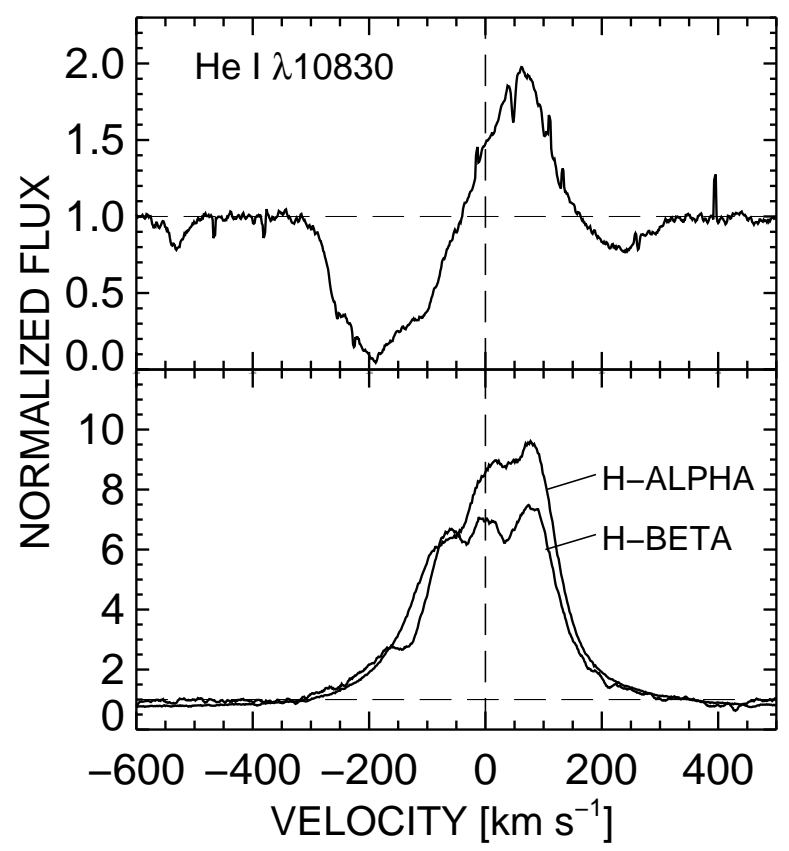

Fig. 7.- The wind-sensitive He I $\lambda 10830$ line taken with PHOENIX on Gemini-S on the same night (JD 2454160.7) and within an hour of the $\mathrm{H} \alpha$ and $\mathrm{H} \beta$ spectra (Dupree et al. 2012). The near-IR helium absorption shows that the wind extends to $\sim-300 \mathrm{~km} \mathrm{~s}^{-1}$, and the emission is weaker than found in previous years. In addition stronger downflow (extending to $+300 \mathrm{~km} \mathrm{~s}^{-1}$ ) than found previously (Dupree et al. 2005) is indicated by absorption on the positive velocity side of the profile. At the same time, such high velocities are not visible in the Balmer series, although the wind clearly modifies these lines at lower velocities $\left(0\right.$ to $\left.-200 \mathrm{~km} \mathrm{~s}^{-1}\right)$. The $\mathrm{H} \alpha$ profile is reduced for display in this figure by a factor of 4 . 